Z. Phys. Chem. 217 (2003) 1031-1044

(C) by Oldenbourg Wissenschaftsverlag, München

\title{
Prediction of Reduced Falloff Curves for Recombination Reactions at Low Temperatures
}

\author{
By Carlos J. Cobos ${ }^{1}$ and Jürgen Troe ${ }^{2, *}$ \\ 1 INIFTA, Facultad de Ciencias Exactas, Universidad Nacional de La Plata, Argentina \\ 2 Institut für Physikalische Chemie, Universität Göttingen, Tammannstrasse 6, \\ D-37077 Göttingen, Germany
}

Dedicated to Prof. Dr. Dr. h.c. mult. Friedrich Hensel on the occasion of his $70^{\text {th }}$ birthday

(Received March 3, 2003; accepted March 18, 2003)

\section{Recombination Reactions / Falloff Curves / Reaction Kinetics / Atmospheric Chemistry}

Strong collision falloff curves for barrierless recombination reactions at low temperatures are calculated taking into account transitional modes only. Specific rate constants $k(E, J)$ from statistical adiabatic channel/classical trajectory (SACM/CT) calculations are used. Broadening factors of the falloff curves are found to depend only weakly on the temperature. A systematic analysis of the influence of the centrifugal barriers $E_{0}(J)$, which are governed by the potential energy surface of the bond energies $E_{0}$, and of the number of transitional modes is made. Guidelines for estimating center broadening factors and shape functions for the broadening factors are given.

\section{Introduction}

Falloff curves of unimolecular dissociation (or isomerization) and the reverse radical recombination reactions are of considerable importance in atmospheric chemistry, in combustion, and in other applications of gas phase kinetics. They characterize the pressure dependence of the rate coefficients $k$ between the low pressure and high pressure limiting ranges. E.g., for radical recombination reactions following the energy transfer mechanism in its Lindemann version

$$
\begin{aligned}
\mathrm{A}+\mathrm{B} & \rightarrow \mathrm{AB}^{*} \\
\mathrm{AB}^{*} & \rightarrow \mathrm{A}+\mathrm{B} \\
\mathrm{AB}^{*}+\mathrm{M} & \rightarrow \mathrm{AB}+\mathrm{M},
\end{aligned}
$$

\footnotetext{
* Corresponding author. E-mail: shoff@gwdg.de
} 
the pseudo-second order rate coefficient

$$
k=([\mathrm{A}][\mathrm{B}])^{-1} \mathrm{~d}[\mathrm{AB}] / \mathrm{d} t
$$

can be represented by

$$
\frac{k}{k_{\infty}}=\frac{k_{0} / k_{\infty}}{1+k_{0} / k_{\infty}}
$$

with the limiting low pressure rate coefficient

$$
k_{0}=\left(k_{1} / k_{-1}\right) k_{2}[\mathrm{M}]
$$

and the limiting high pressure rate coefficient

$$
k_{\infty}=k_{1} .
$$

Eq. (4), i.e. the reduced falloff expression of the Lindemann model, has a particularly simple form. Unfortunately, reality is more complicated. The energy $E$ - and angular momentum (quantum number $J$-) dependences and the multistep character of the reactions (1), (-1), and (2) result in broadenings of the reduced falloff expressions [1] which can be quantified by broadening factors $F\left(k_{0} / k_{\infty}\right)$ to be multiplied with Eq. (4),

$$
\frac{k}{k_{\infty}}=\frac{k_{0} / k_{\infty}}{1+k_{0} / k_{\infty}} F\left(k_{0} / k_{\infty}\right) .
$$

These may be separated into weak collision broadening factors $F^{\mathrm{WC}}\left(k_{0} / k_{\infty}\right)$, related to the weak collision efficiency $\beta_{\mathrm{c}}$ [1], and strong collision broadening factors $F^{\mathrm{SC}}\left(k_{0} / k_{\infty}\right)$, obtained for strong collisions where $\beta_{\mathrm{c}}=1$,

$$
F\left(k_{0} / k_{\infty}\right)=F^{\mathrm{WC}}\left(k_{0} / k_{\infty}\right) F^{\mathrm{SC}}\left(k_{0} / k_{\infty}\right) .
$$

Often the rate coefficients $k$ need to be implemented into large modelling schemes with hundreds of reaction steps such that the simplest possible representation of $k_{0}, k_{\infty}$, and $F\left(k_{0} / k_{\infty}\right)$ is required. With this aim broadening factors first were extracted from Kassel integrals and from rigid activated complex RRKM theory [1]-[3]. Relatively simple functional forms of $F\left(k_{0} / k_{\infty}\right)$ were derived which, to a first approximation, were represented by

$$
F\left(k_{0} / k_{\infty}\right) \approx F_{\text {center }}^{1 /\left\{1+\left[\log \left(k_{0} / k_{\infty}\right) / N\right]^{2}\right\}}
$$

with

$$
N \approx 0.75-1.27 \log F_{\text {center }}
$$


$\left(\log =\log _{10}\right)$. In addition, the center broadening factors $F_{\text {center }}$ were expressed as functions of the Kassel parameters $S_{\mathrm{K}}$ and $B_{\mathrm{K}}$ which were related to the ratios $U_{\mathrm{vib}}^{\neq} / k T\left(S_{\mathrm{K}}=1+U_{\mathrm{vib}}^{\neq} / k T, U_{\mathrm{vib}}^{\neq}=\right.$internal energy of the activated complexes) and $E_{0} / k T\left(B_{\mathrm{K}} \propto E_{0} / k T\right.$, see below, $E_{0}=$ bond dissociation energy of $\mathrm{AB})$. Weak collision broadening factors, in addition, were related to the collision efficiency $\beta_{\mathrm{c}}[4]-[6]$.

Eqs. (7)-(10) have become quite popular in practical applications, mostly using $F_{\text {center }}$ as an empirical fit parameter, although deviations from the shape function of Eq. (9) were realized, particularly for higher temperatures. There are shifts of the minimum of $F\left(k_{0} / k_{\infty}\right)$, asymmetries and deviations from of Eq. (10) [1]-[3]. Some of these defects were eliminated by more complicated functional forms of $F\left(k_{0} / k_{\infty}\right)$ such as proposed in [1]-[13]. However, not much is gained by these modifications because rigid activated complex RRKM theory has its problems with barrierless radical recombination and the reverse simple bond fission reactions. In addition, collisional energy transfer, which also effects reduced falloff curves, is understood only incompletely. It is the aim of the present article to improve reduced falloff expressions for barrierless radical recombination reactions at low temperatures where the conventional RRKM approach is most problematic. Improvements of weak collision broadening factors, which also are required, are not considered here.

\section{General formalism}

Reduced falloff curves of recombination and the reverse dissociation reactions are identical. We, therefore, start from the conventional strong collision rate coefficient $k_{\text {diss }}$ of a unimolecular reaction, see $e . g$. [2]

$$
k_{\text {diss }}=\sum_{J=0}^{\infty}(2 J+1) \int_{E_{0}(J)}^{\infty} \frac{k(E, J) f(E, J) Z[\mathrm{M}]}{k(E, J)+Z[\mathrm{M}]} \mathrm{d} E
$$

which accounts for the $E$ - and $J$-dependence of the specific rate constants $k(E, J)$, replacing $k_{-1}$, and the $J$-dependence of the dissociation threshold energy $E_{0}(J) . f(E, J)$ denotes the equilibrium populations and $Z$ the overall collisional energy transfer frequency. $f(E, J)$ includes the rovibrational density of states $\rho(E, J)$. Following statistical unimolecular rate theory, $k(E, J)$ is expressed by

$$
k(E, J)=W(E, J) / h \rho(E, J)
$$

with the number of open channels (or "activated complex states") $W(E, J)$. It can be shown [3] that the strong collision broadening factors, corresponding to Eq. (11), which at the same time apply to dissociation and recombination 
reactions, then take the form

$$
\begin{aligned}
F^{\mathrm{sC}}\left(k_{0} / k_{\infty}\right)= & (1+x) \sum_{J=0}^{\infty}(2 J+1) \int_{E_{0}(J)}^{\infty} \mathrm{d}(E / k T)\left[F_{\rho} F_{W} /\left(x F_{\rho}+F_{W}\right)\right] \\
& \times \exp (-E / k T)
\end{aligned}
$$

with

$$
\begin{aligned}
& x=k_{0} / k_{\infty}, \\
& F_{\rho}=\frac{\rho(E, J)}{\sum_{J=0}^{\infty}(2 J+1) \int_{E_{0}(J)}^{\infty} \mathrm{d}(E / k T) \rho(E, J) \exp (-E / k T)},
\end{aligned}
$$

and

$$
F_{W}=\frac{W(E, J)}{\sum_{J=0}^{\infty}(2 J+1) \int_{E_{0}(J)}^{\infty} \mathrm{d}(E / k T) W(E, J) \exp (-E / k T)} .
$$

$W(E, J)$ contains contributions from "conserved modes" and "transitional modes". The conserved modes are the vibrational (and internal rotational) modes of the separated A and B. Most of these modes have such high frequencies that they do not contribute to low temperature applications such as considered in the present work. We, therefore, in the present work neglect such modes. Their contributions to $F^{\mathrm{SC}}\left(k_{0} / k_{\infty}\right)$ have extensively been investigated in [1]-[3]. Instead, in $W(E, J)$ we only include transitional modes which are of rotational character, behave classically down to very low temperatures, and have not been investigated systematically in their influence on $F^{\mathrm{SC}}\left(k_{0} / k_{\infty}\right)$ before. The $J$-dependence of these modes is not properly accounted for by conventional RRKM theory while phase space theory (PST) is much more appropriate although it neglect effects due to the anisotropy of potential energy surfaces. We base our approach on recent statistical adiabatic channel/classical trajectory calculations (SACM/CT) [14] which account for angular momentum couplings and anisotropy effects of the potentials. Applications to the reaction $\mathrm{H}+\mathrm{O}_{2} \rightarrow \mathrm{HO}_{2}$ on an ab initio potential have been demonstrated before [15] while falloff curves for the reaction $\mathrm{HO}+\mathrm{NO}_{2} \rightarrow \mathrm{HONO}_{2}$ have been determined with a more empirical potential in [16]. In the following, we do a systematic analysis of falloff curves for model potentials which are constructed as modifications of the $\mathrm{HONO}_{2}$ potential. It is our aim to compare the results with Eqs. (9) and (10) and to provide more accurate alternatives for low temperature systems which are governed by transitional modes only. 


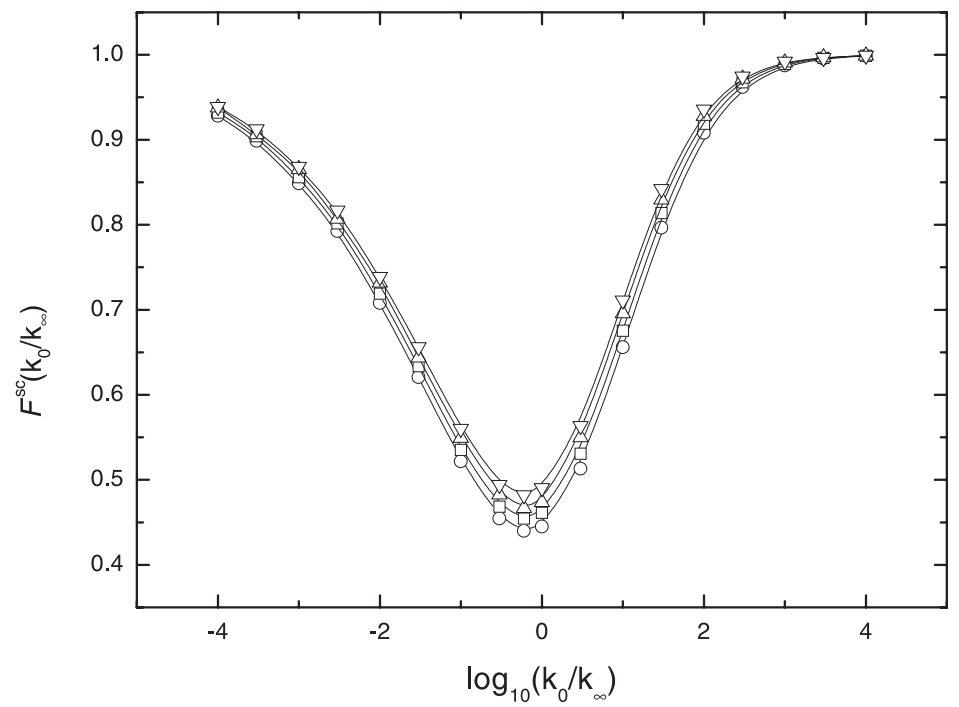

Fig. 1. Strong collision broadening factors for the recombination reaction $\mathrm{HO}+\mathrm{NO}_{2}$ $(+\mathrm{M}) \rightarrow \mathrm{HONO}_{2}(+\mathrm{M})$ at $T=100(\mathrm{O}), 200(\square), 300(\triangle)$, and $400 \mathrm{~K}(\nabla)$.

\section{Broadening factors for varying molecular parameters}

We start from a prototype falloff curve such as calculated for the reaction $\mathrm{HO}+$ $\mathrm{NO}_{2} \Leftrightarrow \mathrm{HONO}_{2}$ in [16]. The molecular parameters of this system have been given in [16] and falloff curves were calculated on the basis of Eqs. (13)(16). The density of states $\rho(E, J)$ was calculated in Whitten-Rabinovitch approximation, treating $\mathrm{HONO}_{2}$ as a spherical top, and implementing anharmonicity corrections following the method from [17]. The number of open channels $W(E, J)$ was expressed by PST, representing $\mathrm{HO}$ as a linear reactant and $\mathrm{NO}_{2}$ as a spherical top and using a capture probability $w_{\text {cap }}(E, J) \approx$ $\left(1-E_{0}(J) / E\right)^{2}$, and accounting for the anisotropy of the potential by multiplying the result with a rigidity factor $f_{\text {rigid }}(E, J) \approx f_{\text {rigid }}(J=0)\left[1-E_{0}(J) / E\right]$, such as suggested by the SACM/CT treatment of [14]. The centrifugal barriers of the system were expressed in the form

$$
E_{0}(J) \approx C_{v}[J(J+1)]^{v}
$$

with $v=1.29$ and $C_{v}=3.95 \times 10^{-3} \mathrm{~cm}^{-1} h c$. While broadening factors were illustrated in [16] over the temperature range $50-1400 \mathrm{~K}$, here we focus attention on the range $100-400 \mathrm{~K}$ only.

Fig. 1 shows broadening factors for the $\mathrm{HO}+\mathrm{NO}_{2}$-system from 100 to $400 \mathrm{~K}$. There is only a weak temperature dependence, showing a slight increase of $F_{\text {center }}^{\mathrm{SC}}$ with increasing temperature. The corresponding rise of $F_{\text {center }}^{\mathrm{SC}}$ with decreasing $B_{\mathrm{K}}$ has also been documented in falloff calculations based on 


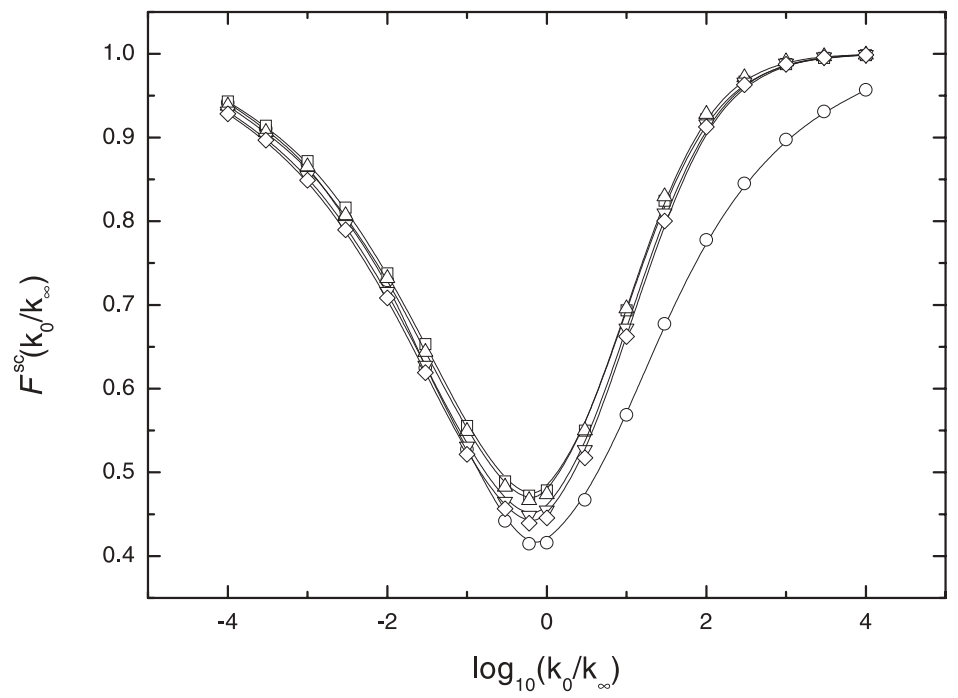

Fig. 2. Strong collision broadening factors, as Fig. 1, for $T=300 \mathrm{~K}$ : variation of centrifugal barriers with $C_{v}$ from Eq. (17) multiplied by $1 / 16(\bigcirc), 1 / 4(\square), 1(\triangle), 4(\nabla)$, and $16(\diamond)$.

Kassel integrals or RRKM theory [1]-[3]. However, the effect is very weak at low temperatures and can practically be neglected. While Fig. 1 corresponds to centrifugal barriers derived from a Morse potential between $\mathrm{HO}$ and $\mathrm{NO}_{2}$ with an estimated Morse parameter $\beta=2.04 \AA^{-1}$, calculations were also made with $\beta=2.6 \AA^{-1}$ from the DFT calculations of [18] and with $\beta=4.18 \AA^{-1}$ from coupled cluster calculations which include single, double and perturbative estimates of triple excitations [19]. The results practically coincided with Fig. 1 and, therefore, are not shown here. In order to see some effect, larger variations of the centrifugal barriers were required. Fig. 2 demonstrates the effects on $F^{\mathrm{SC}}\left(k_{0} / k_{\infty}\right)$ of such variations, changing $C_{v}$ between $1 / 16$ and 16 times the value of $C_{v}$ from Eq. (17). The effects are still small, except for the choice of the smallest $C_{v}$. It should also be mentioned that omitting the rigidity factors, accounting for the anisotropy of the potential, had hardly any effect. While the absolute values of $k_{0}$ and $k_{\infty}$ certainly depend on such details of the potential, the reduced falloff curves do not show dependences to a noticeable extent such that PST in this case appears sufficient to calculate broadening factors.

Changes of the bond energy $E_{0}$ lead to changes of the Kassel parameter $B_{\mathrm{K}}$ and hence to the values of $F_{\text {center }}^{\mathrm{SC}}$, analogous to the temperature effects shown in Fig. 1. Fig. 3 demonstrates the corresponding effects by varying $E_{0}$ between $1 / 4$ and 4 times the value of the $\mathrm{HO}+\mathrm{NO}_{2}$-system. While $4 E_{0}$ approaches the large $B_{\mathrm{K}}$-lower limit of $F_{\text {center }}^{\mathrm{SC}}$, decreasing $B_{\mathrm{K}}$ leads to continuously increasing values of $F_{\text {center }}$. Nevertheless, the effect is not too pronounced. 


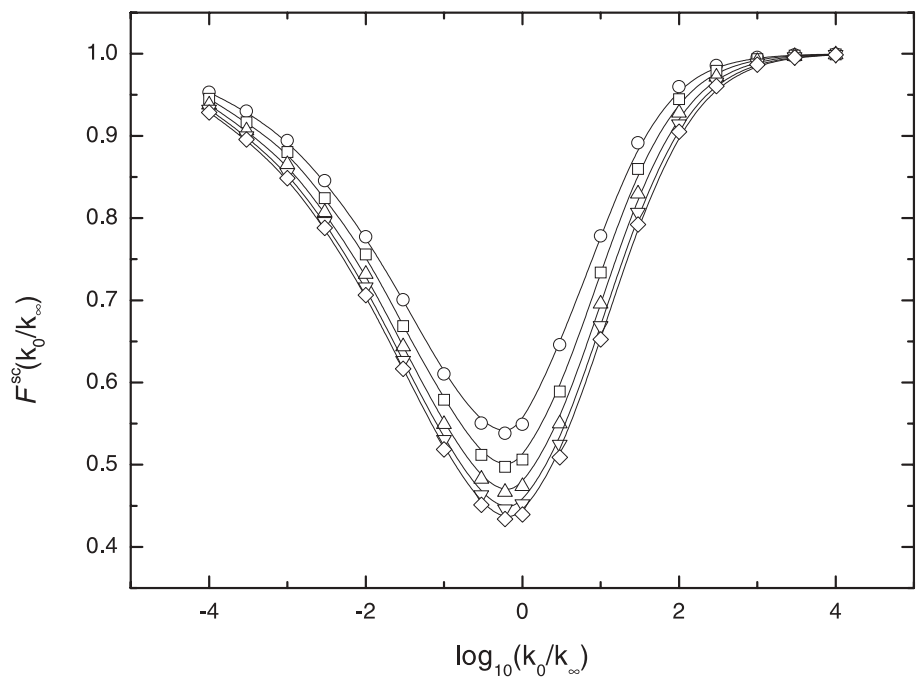

Fig. 3. Strong collision broadening factors, as Fig. 1, for $T=300 \mathrm{~K}$ : variation of bond energies with $E_{0} / h c=16500 \mathrm{~cm}^{-1}$ multiplied by $1 / 4(\bigcirc), 1 / 2(\square), 1(\triangle), 2(\nabla)$, and $4(\diamond)$.

Larger changes of $F_{\text {center }}^{\mathrm{SC}}$ are expected when the number of transitional modes of the reaction changes. Using again the parameters of the $\mathrm{HO}+\mathrm{NO}_{2}$ system as the starting point, we have changed the rotational character of the reactants $\mathrm{A}$ and $\mathrm{B}$ in the recombination reaction (1). Atomic, linear and spherical top A and/or B have been considered. Fig. 4 shows the decrease of $F_{\text {center }}^{\mathrm{SC}}$ with increasing total number $r$ of the rotational degrees of freedom of A and B. $F_{\text {center }}^{\mathrm{SC}}$ decreases from 0.63 for $r=2$ to 0.41 for $r=6$. It was shown in [15] that anharmonicity and anisotropy had some influence on $F_{\text {center }}^{\mathrm{sC}}$ for the $\mathrm{H}+\mathrm{O}_{2}-$ system, i.e. for $r=2$ where $F_{\text {center }}^{\mathrm{SC}}(300 \mathrm{~K}) \approx 0.73$. Such effects, however, were much less pronounced for systems with $r>2$ and are not further considered here.

The numerical results of our calculations are documented in Table 1 where values of $F_{\text {center }}^{\mathrm{SC}}$ are given for a series of calculations such as illustrated in Figs. 1-4.

\section{Functional shapes of broadening factors}

While the symmetric function of Eq. (9) provided a satisfactory representation of $F^{\mathrm{SC}}\left(k_{0} / k_{\infty}\right)$ for rigid activated complexes and situations where $F_{\text {center }}^{\mathrm{sC}}$ was not too small, the present systems with transitional modes of rotational character show some deviations from Eq. (9). In this section these deviations are further analyzed. First, there is a shift of the center of the broadening factors from 


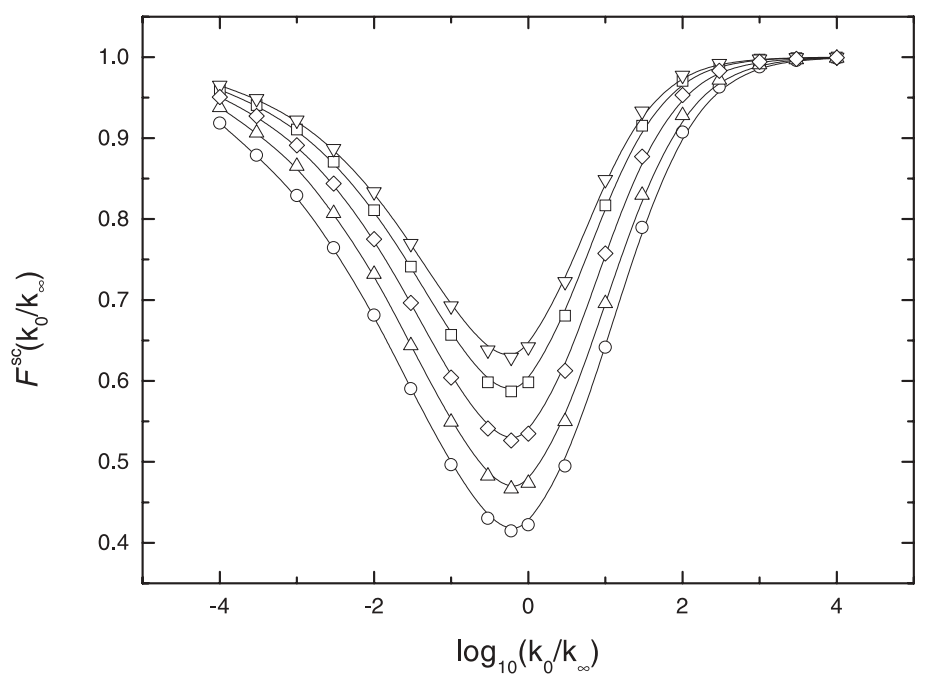

Fig. 4. Strong collision broadening factors, as Fig. 1, for $T=300 \mathrm{~K}$ : change of the structure of the reactants $\mathrm{A}+\mathrm{B} \rightarrow \mathrm{AB}$ with $\mathrm{A}+\mathrm{B}=$ atom + linear $(\nabla)$, atom + spherical top $(\square)$, linear + linear $(\diamond)$, linear + spherical top $(\triangle)$, spherical top + spherical top $(O)$.

Table 1. Strong collision center broadening factors $F_{\text {center }}^{\text {sC }}$ for the recombination reaction $\mathrm{HO}+\mathrm{NO}_{2}(+\mathrm{M}) \rightarrow \mathrm{HONO}_{2}(+\mathrm{M})\left(\right.$ center at $\log \left(k_{0} / k_{\infty}\right) \approx-0.2$; a: normal molecular parameters; b: variation of centrifugal barriers, Eq. (17); c: variation of $\mathrm{HO}-\mathrm{NO}_{2}$ bond energy; $\mathrm{d}$ : variation of molecular complexity of $\mathrm{A}$ and $\mathrm{B}$ in $\mathrm{A}+\mathrm{B} \rightarrow \mathrm{AB}, r=2-6$ is the total number of rotational degrees of freedom of $\mathrm{A}$ and/or B).

\begin{tabular}{lccccc}
\hline & & $T / \mathrm{K}=100$ & 200 & 300 & 400 \\
\hline normal & $\mathrm{a}$ & 0.440 & 0.455 & 0.467 & 0.482 \\
$C_{v} / 16$ & $\mathrm{~b}$ & 0.439 & 0.424 & 0.415 & 0.415 \\
$C_{v} / 4$ & & 0.451 & 0.464 & 0.472 & 0.478 \\
$C_{v}$ & & 0.440 & 0.455 & 0.467 & 0.482 \\
$4 C_{v}$ & & 0.431 & 0.440 & 0.448 & 0.458 \\
$16 C_{v}$ & & 0.428 & 0.434 & 0.439 & 0.445 \\
$E_{0} / 4$ & $\mathrm{c}$ & 0.469 & 0.503 & 0.538 & 0.573 \\
$E_{0} / 2$ & & 0.452 & 0.477 & 0.497 & 0.523 \\
$E_{0}$ & & 0.440 & 0.455 & 0.467 & 0.482 \\
$2 E_{0}$ & & 0.431 & 0.440 & 0.446 & 0.455 \\
$4 E_{0}$ & & 0.426 & 0.431 & 0.434 & 0.439 \\
$r=2$ & $\mathrm{~d}$ & 0.585 & 0.608 & 0.629 & 0.648 \\
\multicolumn{1}{c}{3} & & 0.546 & 0.568 & 0.587 & 0.604 \\
4 & & 0.494 & 0.511 & 0.526 & 0.543 \\
5 & & 0.440 & 0.455 & 0.467 & 0.482 \\
6 & & 0.384 & 0.398 & 0.415 & 0.427 \\
\hline
\end{tabular}




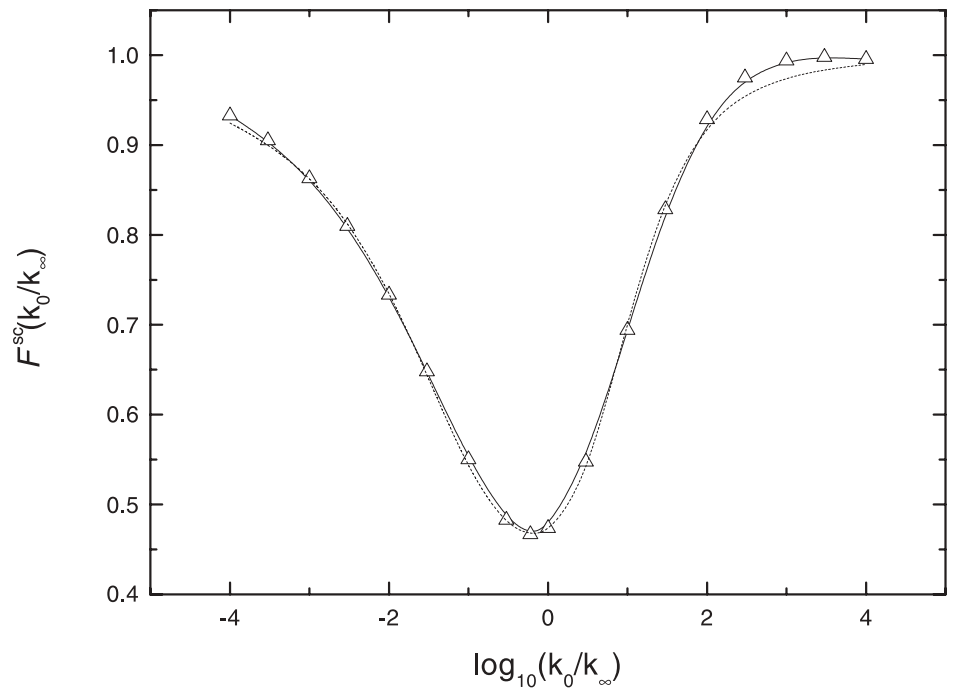

Fig. 5. Fit of strong collision broadening factors from Fig. 1 for $300 \mathrm{~K}(\Delta)$ by Eq. (18) (dotted line) and by Eqs. (19)-(21) (full line), see text.

$x=1$ to $x=0.6$, i.e. from $\log x=0$ to $\log x \approx-0.2\left(\right.$ as before, $\left.x=k_{0} / k_{\infty}\right)$. In addition, the falloff curves are broader at lower pressures $(\log x<-0.2)$ than at higher pressures $(\log x>-0.2)$. Since the present broadening factors for transitional mode-systems all have very similar shapes, it appears worthwhile to look for representations improving Eqs. (9) and (10).

We studied two alternatives. One choice was a function

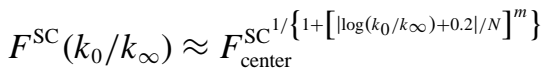

where the parameters $m$ and $N$ had fixed but different values for $\log x<-0.2$ and $\log x>-0.2$. The other choice was a function

$$
F^{\mathrm{SC}}\left(k_{0} / k_{\infty}\right) \approx F_{\text {center }}^{\left.\mathrm{SC}^{1 /}\left\{1+\left[\log \left(k_{0} / k \infty\right)+0.2\right) / N\left(k_{0} / k_{\infty}\right)\right]^{2}\right\}}
$$

where the width $N$ was allowed to vary continuously with $k_{0} / k_{\infty}$.

Fits of the curves from Fig. 1 to Eq. (18) lead to $m=2.34 \pm 0.01$ and $N=1.52 \pm 0.1$ at $\log x<-0.2$ and $m=3.36 \pm 0.05$ and $N=1.17 \pm 0.04$ at $\log x>-0.2$. Fig. 5 demonstrates the good quality of the fit. Similarly good results were obtained for all other examples considered, errors in $F^{\mathrm{SC}}$ being below 0.03 and thus of practically no relevance. Table 2 documents fitted values of $m$ and $N$ for the examples shown in Figs. 1-4.

Expressing the curves from Fig. 1 in the form of Eq. (19) leads to the functions $N\left(k_{0} / k_{\infty}\right)$ which are shown in Fig. 6. The constant value of $N=1.18$ 
Table 2. Fit of shape functions for strong collision broadening factors from Fig. 1 (a): parameters for Eq. (18), subscript - for $\log \left(k_{0} / k_{\infty}\right)<-0.2$, subscript + for $\log \left(k_{0} / k_{\infty}\right)>$ -0.2 ; b) as a) at $T=300 \mathrm{~K}$, variation of molecular complexity, $r=$ total number of rotational degrees of freedom of A and/or B; c) parameters for Eqs. (19), (20) where $N\left(k_{0} / k_{\infty}\right)=\left\{a+b \log \left(k_{0} / k_{\infty}\right)+c\left[\log \left(k_{0} / k_{\infty}\right)\right]^{2}\right\}\left(k_{0} / k_{\infty}\right)^{d}$, see text $)$.

a)

\begin{tabular}{lcccc}
\hline & $T / \mathrm{K}=100$ & 200 & 300 & 400 \\
\hline$m_{-}$ & 2.33 & 2.34 & 2.34 & 2.36 \\
$m_{+}$ & 3.38 & 3.36 & 3.28 & 3.30 \\
$N_{-}$ & 1.53 & 1.52 & 1.51 & 1.52 \\
$N_{+}$ & 1.21 & 1.18 & 1.14 & 1.13 \\
\hline
\end{tabular}

b)

\begin{tabular}{lccccc}
\hline & $r=2$ & 3 & 4 & 5 & 6 \\
\hline$m_{-}$ & 2.41 & 2.39 & 2.36 & 2.34 & 2.30 \\
$m_{+}$ & 3.06 & 3.12 & 3.24 & 3.28 & 3.38 \\
$N_{-}$ & 1.47 & 1.47 & 1.47 & 1.51 & 1.58 \\
$N_{+}$ & 0.94 & 0.98 & 1.07 & 1.14 & 1.19 \\
\hline
\end{tabular}

c)

\begin{tabular}{lrrrr}
\hline & $T / \mathrm{K}=100$ & 200 & 300 & 400 \\
\hline$a$ & 1.486 & 1.463 & 1.423 & 1.421 \\
$b$ & -0.773 & -0.776 & -0.764 & -0.797 \\
$c$ & 0.104 & 0.106 & 0.106 & 0.110 \\
$d$ & 0.178 & 0.179 & 0.177 & 0.180 \\
\hline
\end{tabular}

from Eq. (10), calculated for the average $F_{\text {center }}^{\mathrm{SC}} \approx 0.461$ of the 4 curves in Fig. 1, should be noted for comparison. A fit to $N\left(k_{0} / k_{\infty}\right)$ from Fig. 6 can be made in the form of

$$
\begin{aligned}
& N\left(k_{0} / k_{\infty}\right) \approx\left\{1.448-0.778 \log \left(k_{0} / k_{\infty}\right)+0.1067\left[\log \left(k_{0} / k_{\infty}\right)\right]^{2}\right\} \\
& \times\left(k_{0} / k_{\infty}\right)^{0.178}
\end{aligned}
$$

which, with Eq. (19), gives an excellent fit coinciding with the calculations of Fig. 5. Changes of $N\left(k_{0} / k_{\infty}\right)$ at $300 \mathrm{~K}$ with changing number of rotational degrees of freedom $r$ were only small.

Since Eq. (10) predicts an increase of $N$ with decreasing $F_{\text {center }}^{\mathrm{SC}}$, we try to represent the corresponding changes of Eq. (20) in an analogous way. This is 


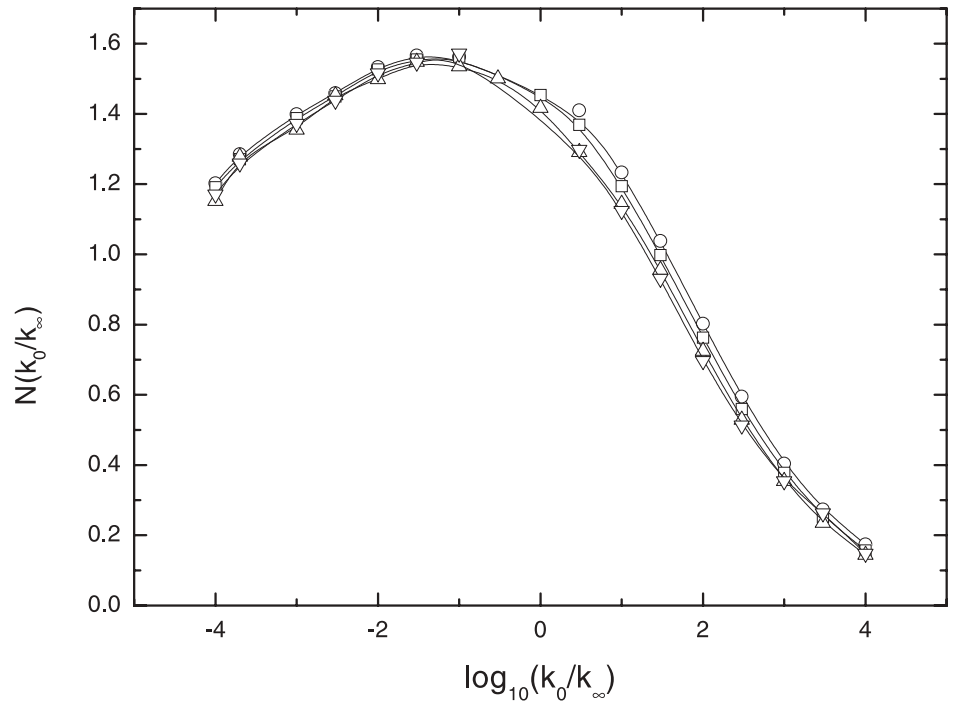

Fig. 6. Width function $N\left(k_{0} / k_{\infty}\right)$ in Eq. (19) for strong collision broadening factors from Fig. 1 (symbols as in Fig. 1).

best done by multiplying Eq. (20) with a factor corresponding to Eq. (10), i.e.

$$
N\left(k_{0} / k_{\infty}\right) \approx \frac{0.75-1.27 \log F_{\text {center }}^{\mathrm{SC}}}{0.75-1.27 \log F_{\text {center }, 0}^{\mathrm{SC}}} N_{0}\left(k_{0} / k_{\infty}\right)
$$

where $N_{0}\left(k_{0} / k_{\infty}\right)$ is given by Eq. (20) and $F_{\text {center }, 0}^{\mathrm{SC}}=0.461$.

\section{Prediction of center broadening factors}

The inspection of Kassel integrals and of rigid activated complex RRKM theory in [1]-[3] allowed to establish relationships between center broadening factors $F_{\text {center }}^{\mathrm{SC}}$ and modified Kassel parameters $S_{\mathrm{K}}$ and $B_{\mathrm{K}}$. These parameters were determined as

$$
S_{\mathrm{K}}=1+\sum_{i=1}^{s-1}\left(h v_{i}^{\neq} / k T\right) /\left[\exp \left(h v_{i}^{\neq} / k T\right)-1\right],
$$

for a molecule $\mathrm{AB}$ with s oscillators and activated complex frequencies $v_{i}^{\neq}$, and

$$
B_{\mathrm{K}}=\left(\frac{S_{\mathrm{K}}-1}{s-1}\right)^{1.6}\left(\frac{E_{0}+a\left(E_{0}\right) E_{z}}{k T}\right)
$$

with the Whitten-Rabinovitch factor $a\left(E_{0}\right)$ and the zeropoint energy $E_{z}$ of AB. With the parameters $S_{\mathrm{K}}$ and $B_{\mathrm{K}}$, useful approximations for $F_{\text {center }}^{\mathrm{sC}}$ were derived 
in the form

$$
-\log F_{\text {center }}^{\mathrm{SC}} \approx \frac{\left(1.06 \log S_{\mathrm{K}}\right)^{2.2}}{1+C_{1} S_{\mathrm{K}}^{C_{2}}}
$$

where

$$
C_{1}=0.10 \exp \left(2.5 B_{\mathrm{K}}^{-1}-0.22 B_{\mathrm{K}}-6 \times 10^{-10} B_{\mathrm{K}}^{6}\right)
$$

and

$$
C_{2}=1.9+4.6 \times 10^{-5} B_{\mathrm{K}}^{2.8} .
$$

More accurate, but also more complicated expressions were derived in [3]. In our present work we have assumed that, for all conserved modes, $h v_{i}^{\neq} \gg k T$ and that the transitional modes out of the $s-1$ modes in Eq. (22) are of rotational character and can be treated classically. One might hope that, in this case, $S_{\mathrm{K}}$ could be identified with $1+r / 2$, then calculate $B_{\mathrm{K}}$ with Eq. (23), and finally derive $F_{\text {center }}^{\mathrm{SC}}$ from Eqs. (24)-(26). We tried to follow this concept but the results were not particularly satisfactory. Instead one may directly use the results from Fig. 4 such as given numerically in Table 2 for $r=2-6$ and $T=300 \mathrm{~K}$. A simple and reasonably accurate representation of these results is also given by

$$
\log F_{\text {center }}^{\mathrm{SC}}=-0.62( \pm 0.05) \log S_{\mathrm{K}}
$$

with $S_{\mathrm{K}}=1+r / 2$ which reproduces the calculated $F_{\text {center }}^{\mathrm{SC}}$ at $300 \mathrm{~K}$ within \pm 0.02 .

For high temperatures, one may try to combine the transitional modes, with a contribution of $r / 2$, and the conserved modes in $S_{\mathrm{K}}$, with a contribution following Eq. (22). It then remains to be investigated whether Eqs. (23)-(26) still work sufficiently well for combined systems of conserved and transitional modes. Likewise, the validity of Eqs. (19)-(21) for asymmetric broadening factors in these systems remains to be checked. This will be the aim of a further investigation [20].

\section{Conclusions}

As a result of the present work, we recommend broadening factors for recombination reactions at low temperatures which are easily estimated. For the center broadening factors we recommend the use of

$$
F_{\text {center }} \approx F_{\text {center }}^{\mathrm{SC}} F_{\text {center }}^{\mathrm{WC}}
$$

with

$$
F_{\text {center }}^{\mathrm{SC}} \approx S_{\mathrm{K}}^{-0.62}
$$


where $S_{\mathrm{K}}=1+r / \underline{2}$ and where $r$ is the total number of external rotational modes of $\mathrm{A}$ and/or $\mathrm{B}$, and with

$$
F_{\text {center }}^{\mathrm{WC}} \approx \beta_{\mathrm{c}}^{0.14},
$$

see [4]-[6]. Eqs. (9) and (10) may provide a first approximation for $F\left(k_{0} / k_{\infty}\right)$ with this $F_{\text {center }}$ which is sufficient for some applications. Better accuracies, however, will be obtained with

$$
F\left(k_{0} / k_{\infty}\right) \approx F_{\text {center }}^{1 /\left\{1+\left[\left(\log \left(k_{0} / k_{\infty}\right)+0.2\right) / N\left(k_{0} / k_{\infty}\right)\right]^{2}\right\}}
$$

where

$$
\begin{aligned}
& N\left(k_{0} / k_{\infty}\right) \approx\left[\frac{0.75-1.27 \log F_{\text {center }}}{1.18}\right] \\
& \times\left\{1.448-0.778 \log \left(k_{0} / k_{\infty}\right)+0.1067\left[\log \left(k_{0} / k_{\infty}\right)\right]^{2}\right\}\left(k_{0} / k_{\infty}\right)^{0.178} .
\end{aligned}
$$

There is a minor temperature dependence of $F_{\text {center }}^{\mathrm{sC}}$ which is not represented by Eq. (29). However, this is practically compensated by the opposite temperature dependence of $F_{\text {center }}^{\mathrm{WC}}$ in Eq. (30) when $\beta_{\mathrm{c}}$ decreases with increasing temperature [1]. We, therefore, recommend to determine $F\left(k_{0} / k_{\infty}\right)$ at $300 \mathrm{~K}$ and use these broadening factors unchanged over the range $100-400 \mathrm{~K}$.

\section{Acknowledgement}

Many helpful discussions with Professor Friedrich Hensel and financial support by the Deutsche Forschungsgemeinschaft (SFB 357 "Molekulare Mechanismen unimolekularer Reaktionen") are gratefully acknowledged.

\section{References}

1. J. Troe, J. Phys. Chem. 83 (1979) 114.

2. J. Troe, Ber. Bunsenges. Phys. Chem. 87 (1983) 161.

3. J. Troe and V. G. Ushakov, Faraday Disc. Chem. Soc. 119 (2001) 145.

4. J. Troe, Ber. Bunsenges. Phys. Chem. 78 (1974) 478.

5. K. Luther and J. Troe, 17th Symp. (Int.) on Combustion, The Combustion Institute, Pittsburgh (1979), p. 535.

6. R. G. Gilbert, K. Luther, and J. Troe, Ber. Bunsenges. Phys. Chem. 87 (1983) 169.

7. I. Oref, J. Phys. Chem. 93 (1989) 3465.

8. Z. Pawlowska and I. Oref, J. Phys. Chem. 94 (1990) 567.

9. Z. Pawlowska, W. C. Gardiner, and I. Oref, J. Phys. Chem. 97 (1993) 5024.

10. H. Wang and M. Frenklach, Chem. Phys. Lett. 205 (1993) 271.

11. A. Kazakov, H. Wang, and M. Frenklach, J. Phys. Chem. 98 (1994) 10598.

12. O. Prezhdo, J. Phys. Chem. 99 (1995) 8633.

13. P. K. Venkatesh, J. Phys. Chem. A 104 (2000) 280. 
14. A. I. Maergoiz, E. E. Nikitin, J. Troe, and V. G. Ushakov, J. Chem. Phys. 117 (2002) 4201.

15. J. Troe, 28th Symp. (Int.) on Combustion, The Combustion Institute, Pittsburgh (2000), p. 1463.

16. J. Troe, Int. J. Chem. Kinet. 33 (2001) 878.

17. J. Troe, Chem. Phys. 190 (1995) 381.

18. D. Chakraborty, J. Park, and M. C. Lin, Chem. Phys. 231 (1998) 39.

19. C. J. Cobos, React. Kinetics Catal. Lett. 79 (2003) 263.

20. C. J. Cobos and J. Troe, to be published. 